\title{
Endoscopic Laser Surgery: Design, Modeling and Control
}

\author{
Rupert Renevier ${ }^{1}$, Brahim Tamadazte ${ }^{1}$, Member, IEEE, Kanty Rabenorosoa ${ }^{1}$, Member, IEEE, Laurent Tavernier ${ }^{2}$, \\ and Nicolas Andreff ${ }^{1}$ Member, IEEE
}

\begin{abstract}
This paper deals with the design, modeling and control of a compact endoscopic tip for laser steering. It consists of a two degrees-of-freedom (DOF) microrobotic device based on two linear piezoelectric motors associated with a deformable microfabricated Silicon mirror. The proposed device is integrated into an endoscopic tip for microrobot-assisted vocal fold laser microsurgery. The laser motions on the target tissue are controlled using the microrobot device and through a path following scheme combined with a visual servoing controller. Indeed, the surgeon defines, using a tablet, a path to be followed automatically by the laser at high frequency and under a predefined fixed velocity. The developed device was validated successfully in both realist testbench and preclinical cadaver trials.
\end{abstract}

Index Terms-Microrobotics, Micro-Electro-Mechanical System (MEMS), Mirror, Piezoelectric Motors, Laser Surgery, Medical Robotics, Laser Microphonosurgery.

\section{INTRODUCTION}

$\mathbf{M}$ ICRORALP was an FP7 European project which focused on the development of an intuitive and flexible endoluminal endoscopy device for automatic laser microphonosurgery, i.e., laser resection and ablation of cysts and cancerous tissues on the vocal folds.

Currently, vocal fold surgery is performed with a stereomicroscope and a surgical laser source, e.g., carbon-dioxide (CO2), thulium, neodymium-doped yttrium aluminum garnet (Nd:YAG), etc. which is placed outside the patient. Therefore, to get a straight-line projection from the laser source to the vocal folds, through the patient's mouth and larynx, the patient must be placed in an extreme extension of the neck. This causes a significant post-operative trauma, often more important than those corresponding to the vocal fold surgery itself [1]. Also, some patients cannot undergo surgery intervention due to a too rigid neck. Moreover, the fact that the laser source is placed outside the larynx, implies a long working distance from the vocal folds causing leveraging effect on the laser beam which is an important source of error on the laser spot position on the vocal folds. Therefore, laser microphonosurgery is restricted for only a few expert surgeons having high dexterity and know-how [2]. Indeed, vocal folds surgery is extremely demanding in terms of accuracy because of the specific tissue to be resected (fragile, viscous, thin,

1 are with FEMTO-ST Institute, AS2M department, Univ. Bourgogne Franche-Comté, CNRS/ENSMM, 24 rue Savary, 25000 Besançon, France, brahim.tamadaztedfemto-st.fr

2 is with Univ. Hospital of Besançon, Univ. Bourgogne FrancheComté, 3 bd Alexandre Fleming, 25030, Besançon, France, ltavernierechu-besancon.fr

Manuscript received xx, 2016; revised xxx, 2016. difficult healing, and lesions which can be less than $1 \mathrm{~mm}$ ) and the necessity to preserve the patient voice [3].

Within the $\mu$ RALP project, different materials were also developed in order to improve patient safety, the surgical procedure accuracy and the surgeon's dexterity and ergonomics. Thus, to control the laser displacements on the vocal folds, the physician defines a desired trajectory (respectively a region for tissue ablation) using a Surgeon-Robot Interface (SRI), consisting of a tactile tablet and a stylus [4]. Thereafter, using this trajectory, it is possible to design an accurate and robust vision-based controller in order to steer automatically the laser spot on the vocal folds [5], [6].

The other challenge concerns the development of the laser steering device. Generally, the device consists of a 2 DOF active or passive miniature mirror. Such laser steering systems are used in a variety of domains: interferometry [7], periscopes [8], optical microscopy, Optical Coherence Tomography (OCT) [9], automotive, Unmanned Aerial Vehicle (UAVs), adaptive optics [10], etc. Steering a laser beam is also widely investigated in industrial purposes: welding [11], micromachining [12] (e.g., microsystems fabrication, semiconductors, glasses, etc.), metrology [13] as well as in free-contact micromanipulation techniques (e.g., laser trapping) [14], [15]. The consequence of this strong demand is the emergence of an active research area concerning the development of actuated mirrors for laser beam steering. Various steering techniques were investigated in the literature: Risley prisms [16], metastable adjustable prism, compact and integrated galvanometers [17], pan-tilt mirror [18], and mirror driven by piezoelectric actuators [19].

Therefore, different technologies are used to design actuated mirrors of different shapes, degrees-of-freedom (dof), size, range of deformation, bandwidth, etc. Recently, another application has emerged, that is laser surgery. Undoubtedly, it is the eye surgery (i.e., ophthalmology) that has the greatest mastery of this new way to perform surgery using a laser source [20].

Laser surgery, especially laser microphonosurgery, imposes some binding specifications such as size, bandwidth and deformation range of the actuated mirror. This is particularly due to the fact that the steering mirror is placed close to the tissue to be resected (respectively, ablated). These requirements increase the technological and scientific challenges both for the design of the actuated mirror and for the control issues of the laser spot displacements onto the tissue. Beside of the very high technology used in the aforementioned laser steering mirrors, none match simultaneously the different specifications of the laser microphonosurgery, especially in terms of accuracy 
$(\leq 100 \mu \mathrm{m})$, stability, bandwidth $(\approx 100 \mathrm{~Hz})$, wide range of deformation $\left(45^{\circ}\right)$ size and biocompatibility.

Our work focuses on the development of a microrobotic device which can equip a flexible endoscopy system directly insertable inside the larynx which brings the laser source at $20 \mathrm{~mm}$ from the vocal folds. The microrobotic device replaces the bulky galvanometric mirrors which equip the current system (e.g., the AcuBlade system) and has to support high power lasers. This paper builds upon our previous work [21], where an optomechatronic proof-of-concept was shown independently from system integration, and [22], [23], where control laws were derived independently from the mechatronic device. The contribution of this paper is to bridge this gap by proposing a fully functional and extensively tested microrobot, fitting into an actual endoscope. It is also fully packaged, since it embeds the optical path and position sensors that were missing in [21]. Therefore, we focus specifically on the optimized design of the different millimetric components which compose the microrobotic device, their integration into a fully functional system, the modeling as well as the control of the final demonstrator in different experimental conditions of use. The first set of experiments consists of a series of validation tests using a realistic testbench in which the vocal folds are replaced by a piece of ham having similarities with the vocal folds in terms of color and aspect. The second set consists of 4 full days of preclinical tests on human cadaver in the Anatomy lab at Université de Franche-Comté, Besançon, FR. The entire endoscopic system embedding the micromechatronic device is inserted by a surgeon into the cadaver larynx placing the distal part (microrobot) in front to the vocal folds (keeping a distance of $20 \mathrm{~mm}$ to $25 \mathrm{~mm}$ to the targeted tissues). These numerous tests have allowed us to validate the updates of the earlier concept in terms of solidity, accuracy, robustness and rapidity.

In the remainder of this paper, Section II gives a detailed description of the different components which compose the developed laser steering device. Section III deals with the integration and the packaging of the proposed microrobot system while Section IV focuses on the mechanical modeling of the microrobotic device as well as the proposed visual servoing scheme. Section V presents the performed experimental validations in both testbench and human cadaver.

\section{MATERIALS AND METHODS}

The objectives of this work are the development of a laser beam steering device which can be integrated into a flexible endoscopic tip whose purpose is the laser microsurgery. Such a microrobotic tip can not only be used for laser microphonosurgery but also for other surgeries e.g., laparoscopy, gastroesophageal, etc. The common specifications of all these surgeries are non-invasiveness, accuracy, rapidity, clinical addedvalue, and versatility. Our endoscopy tip includes;

- a deformable and gilt pan/tilt mirror;

- two Hall-effect submicrometer sensors;

- a visible laser source (passing through a fiber);

- a fixed millimetric right angle prism placed at $20^{\circ}$ relative to the longitudinal axis of the endoscopic system;
- a stereovision system providing $720 \times 576$ pixels $\mathrm{RGB}$ images at $25 \mathrm{fps}$ for the surgeon view;

- home-made high-speed stereovision system based on fiber bundles and GRIN lenses providing $800 \times 600$ monochrome images at $\approx 1000 \mathrm{fps}$ for the vision-based controller.

All these components fit within a compact packaging device measuring only $9 \times 11 \times 42 \mathrm{~mm}^{3}$. Thereafter, the microrobotic device can be inserted directly onto an endoscopic system of less than $18 \mathrm{~mm}$ of external diameter which is completely compatible with laryngoscopy specifications. Below are detailed the different components which compose the proposed microrobotic system.

\section{A. Genesis of the Passive Silicon Mirror Idea}

The first idea of using a Silicon-based pan/tilt mirror was investigated in [21]. In this work, it was demonstrated that using a passive platform can fit with vocal folds laser microsurgery requirements, especially in terms of accuracy, rapidity (i.e., scanning frequency), and large deformations (i.e., tilting angles of $\approx 30^{\circ}$ ). In practice, tilting angles of $30^{\circ}$ provide a scanning area of $20 \times 20 \mathrm{~mm}^{2}$ on the tissue (when the mirror is placed at a distance of $20 \mathrm{~mm}$ to $25 \mathrm{~mm}$ to the vocal folds) representing the average men and women vocal folds size.

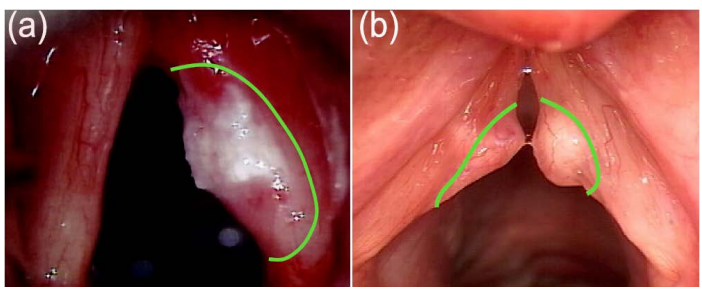

Fig. 1: Initial experimental set-up using a Silicon-based mirror [21].

Furthermore, the compliant structure based on microfabricated Silicon micromirror mirror includes two decoupled miniature platforms controlled independently by pushing on them. Also, platforms are brought back towards their rest position by the springs etched directly onto the Silicon mirror, so the contact is maintained with the actuators. The first experimental validation of this concept was treated in [21] (Fig. 1). In the latter, we validated the general proof of concept of the proposed mirror. However, despite a good behavior of the compliant structure, the obtained performances remained far from the targeted specifications, especially in terms of scanning frequency, maximum tilting angles, and light reflectivity onto the platform. Moreover, the microfabricated mirror was too fragile. Therefore, an optimization study was conducted using the COMSOL multiphysics ${ }^{\circledR}$ in aim to improve the performances of the mirror. Thus, after several optimization processes, from [21] to its final version, the mirror reached the following dimensions $\left(8 \times 8 \times 0.2 \mathrm{~mm}^{3}\right)$, and resonant frequencies of $273 \mathrm{~Hz}$ and $381 \mathrm{~Hz}$ in its internal (pan) and external (tilt) axes, respectively. Moving each axis to its maximum angular position (up to $45^{\circ}$ ) requires a force of $22 \mu \mathrm{N}$ and $65 \mu \mathrm{N}$, respectively. 


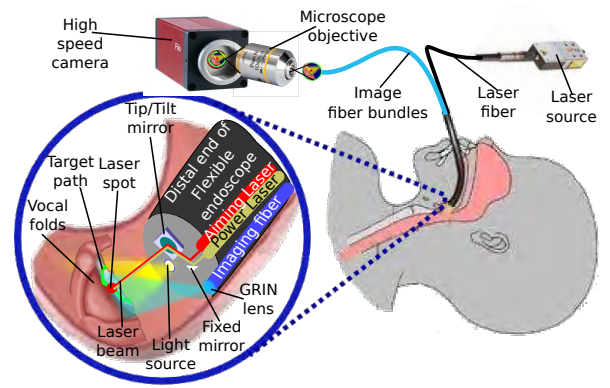

Fig. 2: Silicon on insulator (SOI) based compliant micromirrors.

Also, to ensure a maximum reflectivity of the high power laser (i.e. the power density) arriving onto the mirror, a gold layer is electrodeposited onto the surface (Fig. 2 (a)). It can be highlighted that gold reflects almost $100 \%$ of laser power whose wavelength is superior to $0.7 \mu \mathrm{m}$ (e.g., $\lambda_{\mathrm{CO} 2} \geq 9.4 \mu \mathrm{m}$, $\lambda_{N d-Y A G} \geq 1.06 \mu \mathrm{m}$, etc.). For instance, the developed mirror was tested using a continuous $6 \mathrm{~W}$ Er:YAG laser during 120 seconds.

\section{B. Piezoelectric Micromotors}

As noticed below, the compliant mirror is a passive component, so to steer the laser, it is necessary to push (moving in $z+$ direction) onto each part using a linear stage. Moving in the opposite direction $z-$ is ensured by the microsprings which equip the micromirror (Fig. 2(b)). To do this, we use linear piezoelectric actuators known as Squiggle motors (New Scale Technologies, Inc.). The latter offers a wide range of displacement $(6 \mathrm{~mm})$, high resolution $(0.5 \mu \mathrm{m})$, speed of $7 \mathrm{~mm} / \mathrm{second}$, and a weight of 4.5 grams [24]. All these features are condensed in only a volume of $2.8 \times 2.8 \times 6 \mathrm{~mm}^{3}$ (Fig. 3(a)).

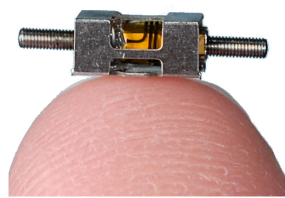

(a)

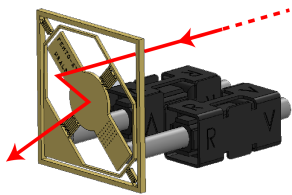

(b)
Fig. 3: (a) Squiggle motor and (b) assembly of the mirror and the motors.

Now, it is possible to assemble the micromirror with the linear micromotors to get the core of the laser steering microrobot (Fig. 3(b)). At this stage, the microrobot can reach an accuracy of $1 \mu \mathrm{m}$ which corresponds to a $0.3 \mathrm{mrad}$ accuracy on each angular axis, thanks to our original design.

\section{Magnetic-based Position Sensors}

Also, two very compact (yet very large compared to the robot itself) magnetic sensors are added to the Squiggle motors in order to get the relative position of the motors. These sensors are based on an array of Hall Effect sensors. They are coupled to a magnetic strip attached to each mobile part

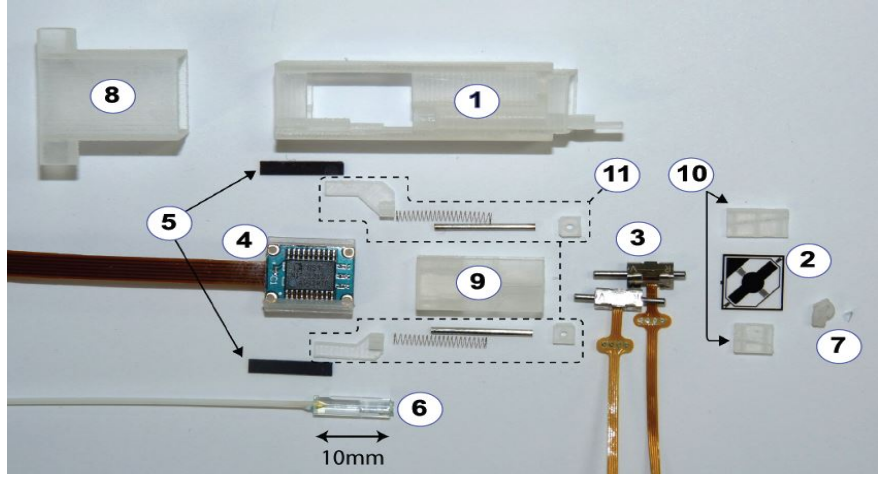

Fig. 4: View on the microrobot individual components before assembly. (1) main frame, (2) compliant SOI mirror, (3) linear piezoelectric motors, (4) magnetic position sensors, (5) magnetic strips, (6) optical fiber, (7) $20^{\circ}$ inclined millimetric right angle prism, (8) housing, (9) actuator fixation, (10), mirror fixation, and (11) magnetic strip holders.

of the linear stages. The sensor array is embedded in a ThinShrink Small Outline Package (TSSOP) close to each sensor. The accuracy of the relative position provided by the sensor is less than $1 \mu \mathrm{m}$ (www.newscaletech.com).

Nevertheless, the challenge is the integration of the sensors in the limited available space, and especially to have the magnetic strip position linked to the shaft linear position. Therefore, we opted for attaching the magnetic strip to a dedicated sub-assembly placed behind the motors, due to lateral space restrictions.

\section{MICROROBOT PACKAGING}

\section{A. Before Assembly}

In addition to the numerous devices mentioned above, the microrobot system includes several small (ranging from some hundred micrometers to some millimeters) components. The latter are necessary to be able to assemble an operating laser steering system. For instance, the developed prototype includes an optical fiber (i.e., laser guide), a millimetric right angle prism placed at $20^{\circ}$ with respect to the longitudinal axis of the endoscopy system, springs for position sensors and preloads, protections, fixations systems, wires, different housing, etc. Finally, not less than 21 parts are required to build the miniaturized laser steering device (Fig. 4). It can be highlighted that for the prototype version, most of them were fabricated using a $3 \mathrm{D}$ printer.

\section{B. After Assembly}

One can integrate the different components in less than 30 minutes. Once the microrobot assembled, it is possible to remove and change the Silicon mirror without disassembling the rest of the system. Fig. 5(a) illustrates a Computer Assisted Design (CAD) view of the designed system when the different components are assembled. In comparison, Fig. 5(b) represents a photography of the assembled system (SQUIPABOT 2.0) which measures $1=42 \mathrm{~mm}, \mathrm{~h}=11 \mathrm{~mm}$ and $\mathrm{w}=9 \mathrm{~mm}$. It can be highlighted that the designed device matches perfectly with 


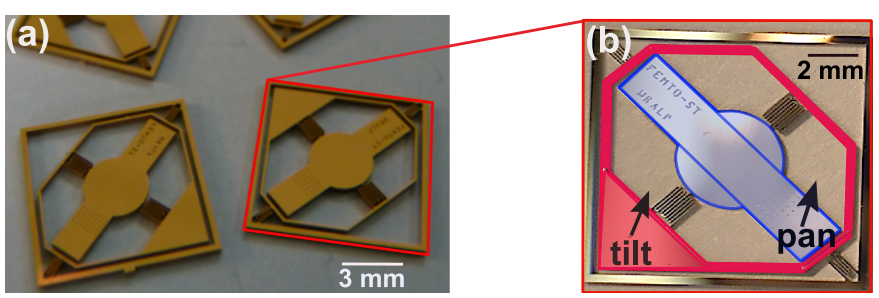

Fig. 5: (a) CAD model of the integrated laser steering system and (b) the assembled microrobot named SQUIPABOT 2.0.

the CAD model design. Due to the dimension of the obtained device, our laser steering can also match with the requirements of laryngoscopy, laparoscopy, etc.

Furthermore, probably the most critical (fragile) part of the developed laser steering system is undoubtedly the SOIbased micromirror which can be changed easily in less than 2 minutes, without removing completely the microrobot from the endoscopic system. However, the system was tested several times for entire days of validation. Indeed, during the cadaver trials, the surgeon had to manipulate the system, without any special precautions, in order to insert the endoscopic system inside the larynx. Nothing was broken, which proves the solidity of our design.

\section{Sterilizability and Biocompatibility Issues}

The microrobot device is packaged inside a printed housing box. Moreover, the cameras and the lighting guides are assembled in the endoscopic tip (circular shape also fabricated using a 3D printer). Thus, the microrobot in turn is inserted completely into the sealed endoscopic tip through its distal part. It can be underlined that all the components are assembled without the use of screws or any other definitive fixing means. Only the mechanical slacks are used to fix the different parts. In effect, the human tissues will never be in contact with the developed microrobotic device, but only with the endoscope outer shell, which can easily be made sterilizable and biocompatible. Therefore, the updated version of the endoscopic system (i.e., the tube) is made using thermoplastic elastomer material. The first thermal sterilization tests of the endoscopic system were successfully performed at the Besançon University Hospital.

\section{MODELING AND CONTROL}

\section{A. Kinematic Modeling}

The optical path followed by the laser beam onto the steering system is schematized in Fig. 6(a). As can be seen, the laser beam comes (via the optical fiber) in the same direction as the longitudinal axis of microrobot because of the anatomic constraint (only $10 \times 10 \mathrm{~mm}^{2}$ are available to embed the microrobot device). Therefore, it is necessary to reflect it on an inclined millimetric right angle prism towards the center of the mirror plate which reflects it towards the target (e.g., vocal folds). Thus, the vision system can visualize a laser spot on the target. The laser spot has from $50 \mu \mathrm{m}$ to $200 \mu \mathrm{m}$ of diameter, perfectly visible by the cameras.

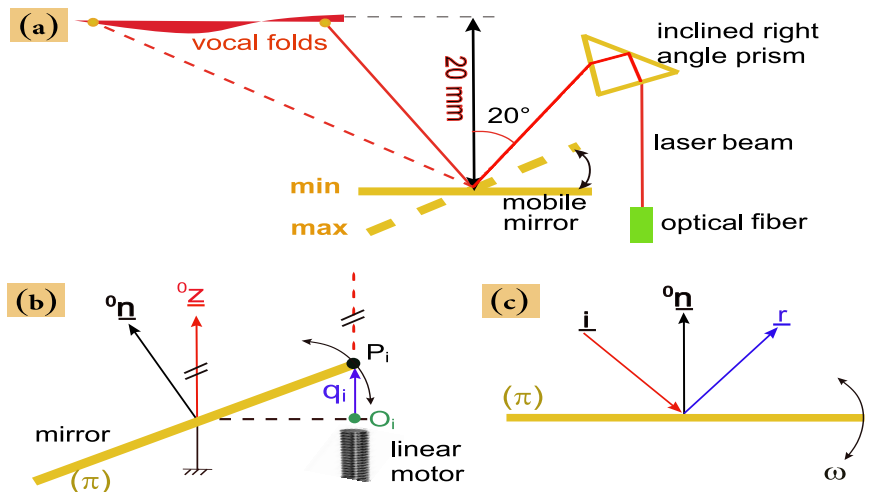

Fig. 6: (a) laser beam path onto the microrobotic system, (b) kinematic modeling of the mobile mirror and (c) the optical path.

Remember that the objectives are to control the laser spot displacements on the vocal folds using different vision-based controllers. The latter consist of closed-loop velocity-based control laws allowing to steer the mirror plate i.e., pan/tilt rotations stages. Consequently, this implies the control of the two linear piezoelectric motors. Let us call $\mathbf{q}_{i}(i \in[1,2])$ the joint position (respectively, $\dot{\mathbf{q}}_{i}$, the corresponding joint velocities) of the linear motors, ${ }^{0} \underline{\mathbf{z}}$ the laser beam direction from the mirror plate towards the target (i.e., vocal folds). Also, by assuming that $\pi$ is the plane representing the central part of the mirror on which the laser reflects, and ${ }^{0} \underline{\mathbf{n}}$ its normal vector. From Fig. 6(b), it is possible to write

$$
\forall \forall^{0} \mathbf{P}_{i} \in \pi: \quad{ }^{\mathbf{0}}{ }^{\top}{ }^{\mathbf{0}} \mathbf{P}_{i}=0
$$

where ${ }^{0} \mathbf{P}_{i}$ represents contact point $i$ between linear motor $i$ and the SOI mirror part $i$. Thereby, ${ }^{0} \mathbf{P}_{i}$ can be formalized by

$$
{ }^{0} \mathbf{P}_{i}={ }^{0} \mathbf{O}_{i}+\mathbf{q}_{\mathbf{i}}{ }^{\mathbf{0}} \underline{\mathbf{z}}
$$

with ${ }^{0} \mathbf{O}_{i}$ the origin of the contact point.

Then, using (1) and (2), it becomes possible to define the following implicit geometric model

$$
{ }^{0} \underline{\mathbf{n}}^{\top 0} \mathbf{O}_{i}+\mathbf{q}_{\mathbf{i}}{ }^{\mathbf{0}} \underline{\mathbf{n}}^{\top \mathbf{0}} \underline{\mathbf{z}}=0
$$

The time-derivation of (3) gives the differential implicit kinematic model of the system. It is defined by

$$
{ }^{0} \underline{\mathbf{n}}^{\top}\left(\mathbf{O}_{i}+\mathbf{q}_{\mathbf{i}}{ }^{\mathbf{0}} \underline{\mathbf{z}}\right)+\dot{\mathbf{q}}_{\mathbf{i}} \underline{\mathbf{n}}^{\top \mathbf{0}} \underline{\mathbf{z}}=0
$$

from which we can extract the differential inverse kinematic model

$$
\dot{\mathbf{q}}_{i}=\frac{-\left(\mathbf{O}_{i}+\mathbf{q}_{\mathbf{i}}^{\top \mathbf{0}} \underline{\mathbf{z}}\right)^{\top}}{{ }^{0} \underline{\mathbf{n}}^{\top}{ }^{0} \underline{\mathbf{z}}} \underline{\dot{\mathbf{n}}}
$$

Finally, to be complete, it is necessary to consider the optical model of the mirror as shown in Fig. 6(c). Therefore, considering the incident laser beam $\underline{\mathbf{i}}$ (linked to the world coordinate system $\mathcal{R}_{0}$ ), and $\underline{\mathbf{r}}$ the reflected beam, one can write

$$
{ }^{0} \underline{\mathbf{n}}=\frac{1}{2}\left(\frac{\underline{\mathbf{r}}-\underline{\mathbf{i}}}{\|\underline{\mathbf{r}}-\underline{\mathbf{i}}\|}\right)
$$

Thereby, time differentiating the latter gives

$$
{ }^{0} \underline{\dot{\mathbf{n}}}=\frac{1}{2}\left(1-{ }^{0} \underline{\mathbf{n}}^{0} \underline{\mathbf{n}}^{\top}\right) \underline{\dot{\mathbf{r}}}
$$


Concatenating (5) and (7) yields the differential kineto-optic model (DKOM):

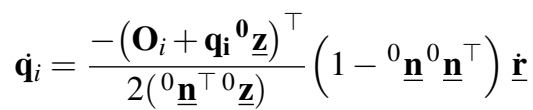

which is the only required model to control the mirror using a vision-based control.

\section{B. Visual Servoing Schemes for Laser Steering}

In previous work, we have developed the theoretical aspects of two different and complementary vision-based controllers for laser spot laser automatic steering. The experimental validation of these controllers were performed using a commercial $25 \times 33 \times 38 \mathrm{~mm}^{3}$ mirror (S-334) from Physical Instruments Inc. The visual servoing control laws are:

- Monocular path following scheme. The originality of the method is based on the decoupling of the path following and the velocity profile which provides a constant velocity on the path regardless of the shape, size or curvature of the incision path [22];

- Stereoscopic point-to-point scheme. It considers two real cameras (stereoscopic vision system) and a virtual camera (obtained by considering the laser source as a virtual camera grabbing one pixel at a time) to construct a trifocal constraint. From the latter, it becomes trivial to design a robust laser steering controller [23].

In the experiments reported below, we only consider the path following method (Fig. 7), which is the most adequate to the laser surgery requirements and to the clinicians' first feedbacks. Also, rather than using the commercial mirror, we use now the developed microrobotic device in close-to-clinical practice.

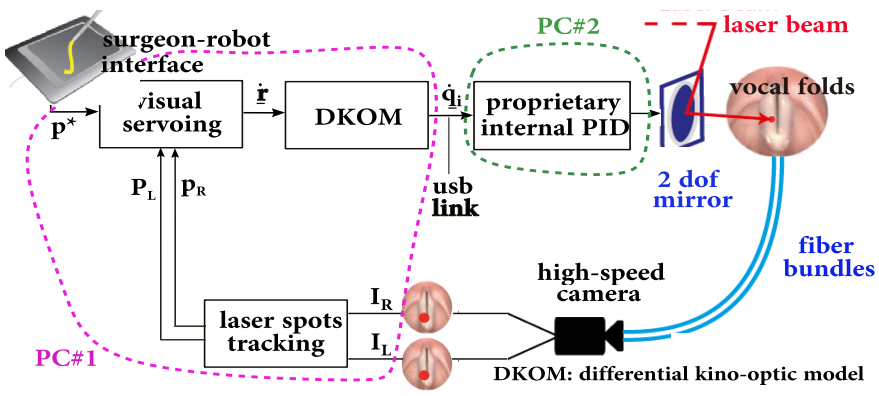

Fig. 7: Control scheme in closed-loop with the robot-surgeon interface (RSI).

\section{EXPERIMENTAL RESULTS}

The experimental validation of the proposed device is performed using two scenarios. Firstly, the microrobotic device is tested using a testbench including two high-speed image bundle systems. The latter is based on two fiber bundles (FUJIKURA FIGH-50-1100N) connected to GRIN (GradientIndex) lens (GRINTECH GT-IFRL-180-inf-50-C1). Each bundle (containing 50,000 fibers) measures $1.2 \mathrm{~mm}$ of diameter and $500 \mathrm{~mm}$ of length. The distal side of each fiber bundle is fixed (using ultra-violet glue) to the GRIN lens. The proximal side is placed near the $\times 10$ microscope objective which equips a high resolution $(2500 \times 1760$ pixels $)$ and high speed camera EoSens (from Mikrotron ${ }^{\circledR}$ ) which can reach $10,000 \mathrm{fps}$ in nominal conditions. The target image is returned to the camera sensor through the mounting "GRIN lens + fiber bundles + objective lens" to the camera sensor. Therefore, the camera sensor views the back-end of both of the bundles in the same image (the full image of $2500 \times 1760$ pixels). In the latter, we crop two sub-images (right and left) of $800 \times 600$ pixels for each one (Fig. 8). Thus, we obtain a synchronized high-speed stereovision system using only one camera. Concerning the laser spot size in the image, it depends on the used lighting source. In this work, we target a laser spot diameter of $50 \mu \mathrm{m}$ to $100 \mu \mathrm{m}$ by tuning the light source power.

Secondly, the steering system is also tested during human cadaver trials by an ENT (Ear, Nose, and Throat) surgeon. Here, SQUIPABOT is inserted in the endoscope developed by our partners at LUH (Leibniz Universität Hannover, DE) [25] and connected to the Surgeon-Robot Interface developed by our other partner IIT (Istituto Italiano di Tecnologia, Genova, IT) [4]. Therefore, instead of using the high-speed vision system, we use two (red-green-blue) RGB MISUMI cameras R (from Misumi Electronics Corp.) characterized with a resolution of $720 \times 576$ pixels and a frame rate of 25 fps.

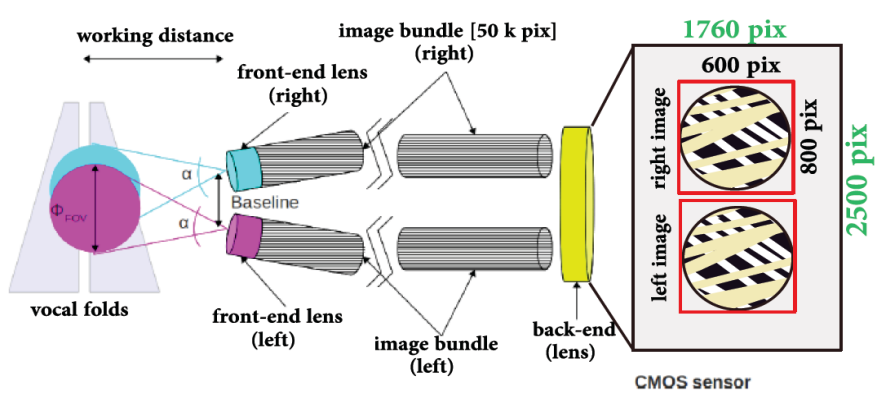

Fig. 8: Concept of the high-speed stereovision system based on fiber bundles and GRIN lenses.

\section{A. Experimentation on a Testbench}

The testbench used in the first scenario is illustrated in Fig. 9. As can be shown the microrobotic structure is packaged inside a $18 \mathrm{~mm}$ outer diameter endoscopy tip. In addition, the latter includes the high-speed stereovision system, the RGB cameras as well as the lighting guides.

\section{B. Path-following Validation}

The path following algorithm developed in [22] is operating as follows: the physician defines a non-parametrized path (i.e., a curve) via a stylet onto a smart tablet [4]. Thus, the freehand drawn path is represented by a set of normalized $\left[x_{T} y_{T}\right]$ points which are sent to the path following algorithm where it will be denormalized in aim to obtain $\left[x_{I} y_{I}\right]$ represented in the image frame. Thereby, the path-following method computes, at each iteration (i.e., each new image), the velocity $\left[\dot{x}_{I} \dot{y}_{I}\right]$ of progress of the laser onto the curve. Thus, the velocities $\left[\dot{x}_{I}\right.$ 


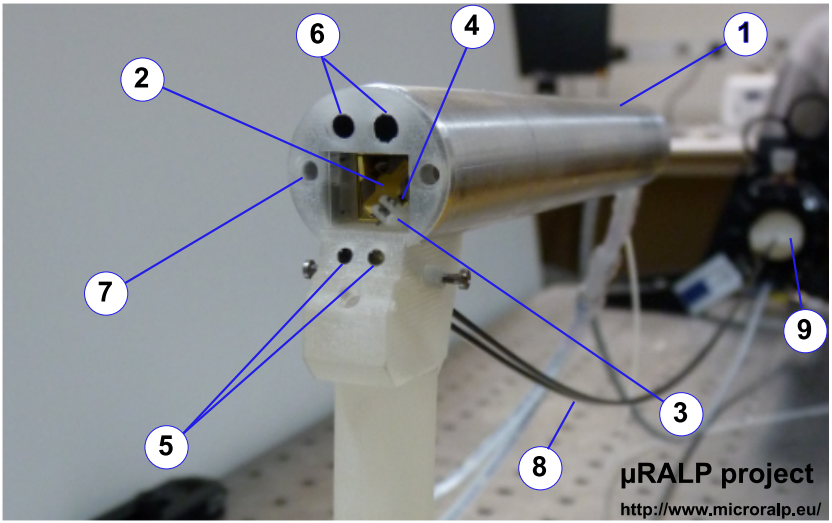

Fig. 9: Final assembled system: (1) endoscopic tube, (2) compliant SOI mirror, (3) inclined millimetric prism, (4) laser fiber, (5) GRIN lens which equip the fiber bundles, (6) RGB cameras, (7) lighting guide, (8) fiber bundles, and (9) EoSens camera.

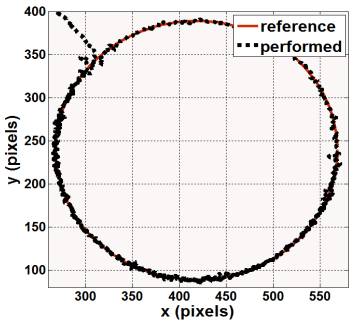

(a)

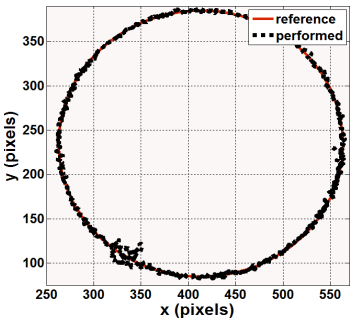

(b)
Fig. 10: Path following achievements using different velocities: (a) $v_{0}=4$ pixels/second, and (b) $v_{0}=6$ pixels $/$ second.

$\left.\dot{y}_{I}\right]$ are transformed using DKOM (8) to the joint velocities $\dot{q}_{i}$ to control the piezoelectric linear stages.

Firstly, the controller is tested several times (using the same velocity of progress) in a simple curve (a circle) in order to quantify its performances in terms of accuracy, rapidity, and reliability. These performances are then compared to the laser surgery specifications in aim to judge the relevance of controller as well as our laser steering system (Fig. 10(a)). A second set of tests are performed using the same curve while varying the velocity $v_{0}$ in order to verify the relevance of the controller for higher velocities (Fig. 10(b)). From this, it can be underlined that the controller qualitatively fits the expectations despite the change of the velocity progress $v_{0}$ and consequently validate the relevance of the developed microrobotic device. The validation tests shown in (Fig. 10) were repeated 30 times in order to access to representative statistics concerning the accuracy.

Table I summarizes the set of tests (each line in the table represents the sum of 3 individual tests). By analyzing each test, it is clear that both microrobotic laser steering device and path-following algorithm demonstrate qualitative performances in term of accuracy: Root Mean Square (RMS) error of $\mathbf{8 0 . 5 0} \mu \mathrm{m}$, standard deviation (STD) of $\mathbf{7 7 . 8 9} \mu \mathrm{m}$. It can be highlighted that $70 \%$ of the tests fits perfectly the laser microphonosurgery specifications (i.e., laser spot position
TABLE I: RMS, STD, $\max _{e}$ and $\min _{e}$ of the path following error in $\mu m$.

\begin{tabular}{|l||c|c||c|c|}
\hline$N^{\circ}$ & RMS $(\mu m)$ & STD $(\mu m)$ & $\max _{e}(\mu m)$ & $\min _{e}(\mu m)$ \\
\hline \hline 1 & 201.55 & 196.54 & 309.01 & 0.004 \\
2 & 40.77 & 30.29 & 153.68 & 0.012 \\
3 & 22.76 & 21.98 & 147.15 & 0.015 \\
4 & 36.86 & 36.57 & 302.43 & 0.019 \\
5 & 39.92 & 38.86 & 155.14 & 0.011 \\
6 & 29.27 & 29.19 & 146.56 & 0.109 \\
7 & 60.49 & 60.22 & 287.70 & 0.029 \\
8 & 43.94 & 42.70 & 153.76 & 0.096 \\
9 & 138.38 & 134.93 & 712.38 & 0.037 \\
10 & 191.13 & 187.68 & 982.29 & 0.136 \\
\hline total & $\mathbf{8 0 . 5 0}$ & $\mathbf{7 7 . 8 9}$ & $\mathbf{3 3 5 . 0 7}$ & $\mathbf{0 . 0 4 6}$ \\
\hline
\end{tabular}

errors $\leq 100 \mu \mathrm{m})$. These statistics were obtained by taking into account, in the error calculation, the distance between the initial laser spot position which not placed directly on the reference curve which increases the path following errors (Fig. 10). Concerning the reliability, as be noticed, the same circle was successfully performed 30 times without any failure and presenting the same order of accuracy. The sampling frequency of the controller or loop during these validation tests is estimate to $\mathbf{4 0} \mathbf{~ H z}$. The latter is mainly limited by the proprietary internal PID loop otherwise the path following controller frequency can reach some hundred hertz.

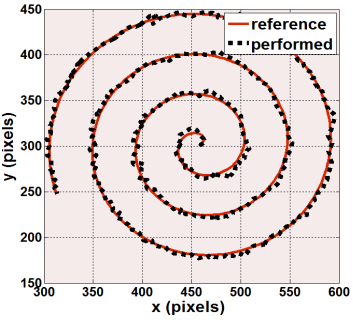

(a)

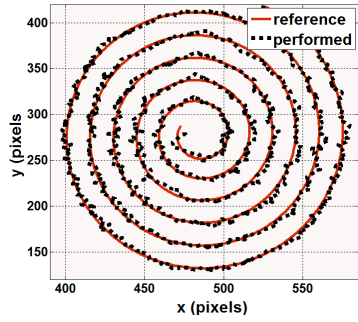

(b)
Fig. 11: Validation on more complex paths (ablation spirals): (a) $v_{0}=2.25$ pixels/second, and (b) $v_{0}=2.25$ pixels $/$ second.

To be closer to the clinical conditions of a laser surgery intervention, we decided to increase the difficulty by taking into account more complex curves (high curvatures) comparing to the laser surgery paths. Thus, the microrobotic system as well as the control law are tested in the curves depicted in Fig. 11. It can be highlighted that our purposes remain operating, with good performances; very similar that those obtained using simple circles as reference paths. More precisely, a RMS error of $32.25 \mu \mathrm{m}(\mathrm{STD}=\mathbf{2 9 . 4 3} \mu \mathrm{m})$ and $\mathbf{3 4 . 3 8} \mu \mathrm{m}(\mathrm{STD}=\mathbf{3 1 . 5 1} \mu \mathrm{m})$ corresponding to the examples depicted in Fig. 11(a) and 11(b), respectively.

\section{Preclinical Validation: Human Cadaver Trials}

The proposed materials and methods were also tested on human cadaver (during 4 full days within 3 months, each day corresponds to an incremental version of the endoscopic system) at the Anatomy lab of "Université de Franche-Comté" under the supervision of Prof. L. Tavernier (ENT surgeon). The cadaver trials consist of the validation of the endoscopy 
system (Fig.12) insertion into the larynx, waterproofing, solidity (e.g., micromechatronics components) and the functioning of the laser steering device as well as the vision-based controller (including the image processing algorithms).

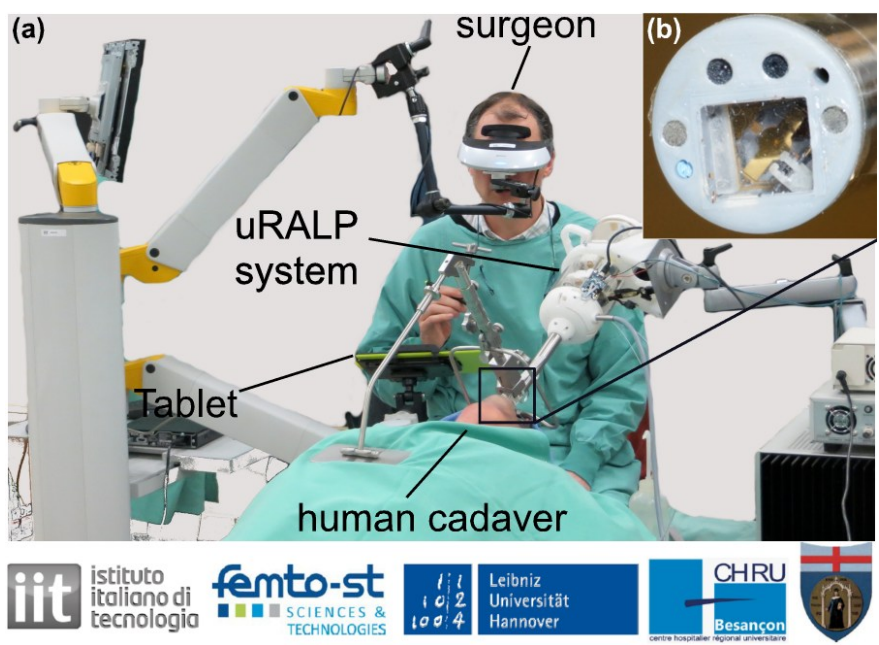

Fig. 12: (a) picture taken during the last $\mu$ RALP project's cadaver trial (picture captured by L. Godard - Université de Franche-Comté) and (b) represents a zoom onto the endoscopic tip (picture captured by A. Schoob - LUH, Hannover, $D E)$.

The endoscopy system [25] containing the microrobot was inserted into the larynx until the distal part (i.e., the microrobot) is in front of the vocal cords i.e., $20-25 \mathrm{~mm}$. Using the smart tablet [4], the surgeon defines a curve on the vocal folds on which the laser should pass.

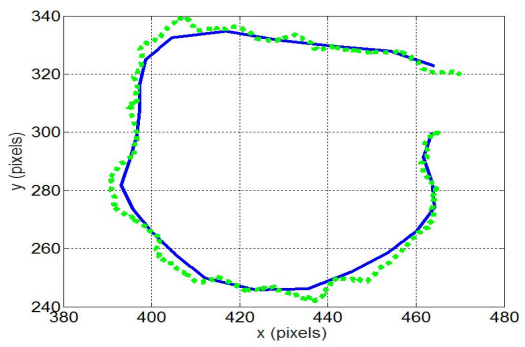

(a) Superposition of a path drawn by the surgeon (in solid blue) and the path followed by the laser (in dashed green)
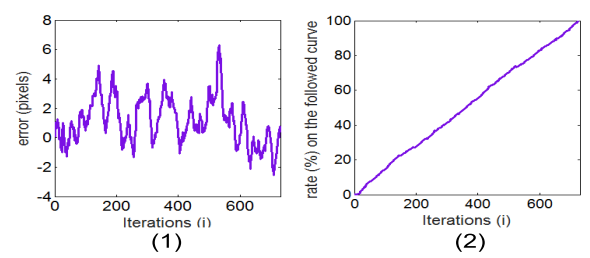

(2)

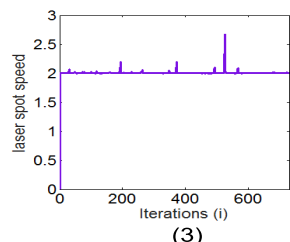

(3) (b) (1) shows the evolution of the errors $v s$. number of iterations, (2) represents the progress rate $(\%)$ of the laser spot onto the path $v s$. number of iterations, and (3) the laser spot velocity $v s$. number of iterations.

Fig. 13: Path following validation on human cadaver.

As shown in Fig. 13(a), the laser follows the reference path with good accuracy (RMS of $\mathbf{8 8 . 3 5} \mu \mathrm{m}$ with an STD of
60.32 $\mu \mathrm{m}$ ) which represents the same order than those obtained on the testbench. Concerning the controller frequency, it decreases to $17 \mathrm{~Hz}$ because of the RGB cameras frame rate limitation. Furthermore, Fig. 13(b-1) shows the error between the reference path and the one carried by the laser using a tablet. In order to illustrate the constant progress velocity of the laser spot on the path drawn by the surgeon, we integrated the laser spot displacements according to the iteration number. As it can be observed, the obtained curve consists of an almost straight line of slope of "1" Fig. 13(b-2) proves that the laser spot velocity is constant as it can be also seen in Fig. 13(b-3).

\section{CONCLUSION}

This paper presented the study, design and validation of a microrobotic device for laser steering. The steering system is based on the use of two miniature linear piezoelectric actuators coupled with a SOI compliant structure and mirror plate. The final assembled device including motors, actuated mirror, fixed prism, optical fiber, sensors, magnetic strips, housing parts, wires, etc., measures: $1=42 \mathrm{~mm}, \mathrm{~h}=11 \mathrm{~mm}$ and $\mathrm{w}=9 \mathrm{~mm}$. Thereby, it can be integrated easily in an endoscopic system. This paper was especially focused on the design, modeling and control of compact and packaged 2 DOF laser steering system. The latter was conceived in order to be easily embedded in an interchangeable endoscopic tip which in turn inserted in an entire endoscopic system of $18 \mathrm{~mm}$ of diameter. Also, two RGB miniature cameras are placed on the endoscopic tip.

Moreover, the developed device was tested and validated in both realistic testbench and preclinical trials (human cadaver trials). The objectives were to assess the relevance and performances of the microrobotic device as well as of the vision feedback controllers (path following) allowing an accurate control of the laser spot onto the vocal folds. Concerning the testbench validation scenario, the path following controller using the microrobot has shown very interesting results in terms of accuracy (RMS error of $80.50 \mu \mathrm{m}$, rapidity of $40 \mathrm{~Hz}$, and reliability (100\% of success rate for 30 individual tests)). As regards preclinical tests, the purposes have presented the same order of relevance (e.g., RMS error of $\mathbf{8 8 . 3 5} \mu \mathrm{m}$ with a rapidity of $17 \mathrm{~Hz}$ ) but at a lower frame rate because of the RGB cameras limits.

The next stages of this work will involve performing more preclinical tests (human cadaver trials) in order to derive more significant evaluation of the microrobot for CE marking approval in view of future clinical trials.

\section{ACKNOWLEDGMENTS}

This work was supported by $\mu$ RALP, the EC FP7 ICT Collaborative Project no. 288663 (www.microralp.eu), and by ACTION, the French ANR Labex no. ANR-11-LABX-0101 (www.labex-action.fr).

The authors would like to thank the partners within the $\mu$ RALP project, with special mention to D. Kundrat (LUH, Hannover, GE) for the numerous helps on the mechanical integration of the microrobot into the $\mu$ RALP endoscopic system. 


\section{REFERENCES}

[1] A. Aloy and M. Grasl, "Endoscopy of larynx and trachea with rigid laryngo-tracheoscopes under superimposed high-frequency jet ventilation (shfjv), ISBN: 978-953-51-1071-2, 2013.

[2] H. Eckel, S. Berendes, M. Damm, and K. JP., "Suspension laryngoscopy for endotracheal stenting," Laryngoscope, vol. 113, pp. 11-15, 2003.

[3] M. Remacle, "Laser-assisted microphonosurgery," in Surgery of Larynx and Trachea, Springer Berlin Heidelberg, 2010, pp. 51-56.

[4] L. Mattos, N. Deshpande, G. Barresi, L. Guastini, and G. Peretti, "A novel computerized surgeon-machine interface for robot-assisted laser phonomicrosurgery," Laryngoscope, vol. 124, p. 1887-1894, 2014.

[5] J.-A. Séon, B. Tamadazte, and N. Andreff, "Path following: from mobile robotics to laser surgery," in 3rd Work. on Visual Control of Mobile Rob. at IEEE/RSJ Int. Conf. on Intel. Rob. and Sys., USA, 2014, pp. 8-15.

[6] B. Tamadazte, and N. Andreff, "Weakly calibrated stereoscopic visual servoing for laser steering: Application to phonomicrosurgery," IEEE/RSJ Int. Conf. on Intel. Rob. and Sys., pp. 743-748, 2014.

[7] S. Mousset, C. Rouyer, G. Marre, N. Blanchot, S. Montant, and B. Wattellier, "Direct measurement of tiled mirror phase difference by multi-wave shearing interferometry," in Conf. on Lasers and ElectroOptics and Conf. on Quantum Elect. and Laser Sci., 2006, pp. 1-2.

[8] S. Rollins, R. Knutson, V. Hung, and S. Ebner, "Autonomous mobile periscope system ," Int. Sym. on Underwater Techn., 1998, pp. 423-427.

[9] S. Samuelson, L. Wu, J. Sun, S. Choe, B. Sorg, and H. Xie, "A 2.8$\mathrm{mm}$ imaging probe based on a high-fill-factor mems mirror and wirebonding-free packaging for endoscopic optical coherence tomography," J. of Microelectromechanical Sys., vol. 21, pp. 1291-1302, 2012.

[10] I. Kanno, T. Kunisawa, T. Suzuki, and H. Kotera, "Development of deformable mirror composed of piezoelectric thin films for adaptive optics," IEEE J. of Selected Topics in Quantum Elect., pp. 155-161, 2007.

[11] M. Fridenfalk and G. Bolmsjo, "Design and validation of a universal 6D seam-tracking system in robotic welding using arc sensing," Adv. Rob., vol. 18, pp. 1-21, 2004.

[12] H. Cui, Z. Xiao, J. Dong, Y. Chen, Z. Hou and G. Zhao, "Towards fast femtosecond laser micromachining of glass, effect of deposited energy," in Int. Conf. on Quality, Reliability, Risk, Maintenance, and Safety Eng., 2013, pp. 2091-2094.

[13] H. Jeon, W. Myeong, J.-U. Shin, J.-W. Park, H.-J. Jung and H. Myung"Experimental Validation of Visually Servoed Paired Structured Light System (ViSP) for Structural Displacement Monitoring," in IEEE/ASME Trans. on Mech., vol. 19, pp. 1603-1611, 2014.

[14] X. LI and C.C. Cheah, "Robotic cell manipulation using optical tweezers with unknown trapping stiffness and limited FOV," in IEEE/ASME Trans. on Mech., vol. PP, pp. 1-9, 2015

[15] Y. Huang, J. Wan, M.-C. Cheng, Z. Zhang, S.-M. Jhiang and C.-H. Menq "Three-axis rapid steering of optically propelled micro/nanoparticles," in Rev. Sci. Instrum., 80(6):063107, 2009.

[16] N. Yamanaka, H. Yamashita, K. Masamune, T. Chiba, T. Dohi "An Endoscope With 2 DOFs Steering of Coaxial Nd:YAG Laser Beam for Fetal Surgery," IEEE/ASME Trans. on Mech., pp. 898-905, 2010.

[17] Z. Zhakypov, E. Golubovic, and A. Sabanovic, "Galvanometric optical laser beam steering system for microfactory application," in Conf. of the IEEE Ind. Elect. Society, 2013, pp. 4138-4143.

[18] T. Sandner, S. Kimme, T. Grasshoff, U. Todt, A. Graf, C. Tulea, A. Lenenbach, and H. Schenk, "Micro-scanning mirrors for high-power laser applications in laser surgery," in Int. Conf. on Optical MEMS and Nanophotonics, 2013, pp. 83-84.

[19] J. Park, H. Lee, S. Yun, Y. Ham, and D. Yun, "Design of a piezoelectricdriven tilt mirror for a fast laser scanner," Japanese J. of Appl. Phys., 2012.

[20] B. Feldman, "Femtosecond laser will not be a standard method for cataract extraction ten years from now," Survey of Ophthalmology, 2015.

[21] K. Rabenorosoa, B. Tasca, A. Zerbib, P. Rougeot, and N. Andreff, T.E. Pengwang, "SQUIPABOT: A Mesoscale Parallel Robot for a Laser Phonosurgery," in Int. J. of Optomechatronics, pp. 310-324, 2015.

[22] J.-A. Séon, B. Tamadazte, and N. Andreff, "Decoupling path following and velocity profile in vision-guided laser steering," IEEE Trans. on Rob., vol. 31(2), pp. 280-289, 2015.

[23] N. Andreff and B. Tamadazte, "Laser steering using virtual trifocal visual servoing," Int. J. of Rob. Res., vol. 35(6), pp. 672-694, 2016.

[24] S. Yang, R.-A. MacLachlan, C.-N. Riviere, "Manipulator Design and Operation of a Six-Degree-of-Freedom Handheld Tremor-Canceling Microsurgical Instrument," IEEE/ASME Trans. on Mech., pp. 761-772, 2015.
[25] D. Kundrat, A. Schoob, L. Kahrs, and T. Ortmaier, "Flexible robot for laser phonomicrosurgery," Soft Rob., pp. 265-271, 2015.

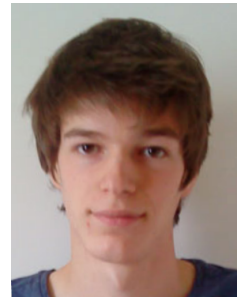

Rupert Renevier received the Engineer degree on Medical Engineering from ENSMM, Besançon, FR, in 2014, and a M.S. degree on Microsystems and Mechatronics from Université de Franche-Comté, Besançon, FR, in 2015. He is currently with the AS2M Department of the FEMTO-ST Institute. His research interests are microrobotic design and control applied to mini-invasive surgery.

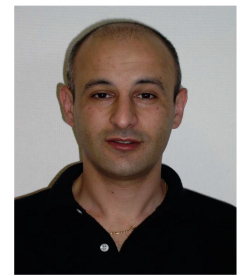

Brahim Tamadazte holds a Ph.D. in Automation and Computer Science from Univ. de FrancheComté in 2009 and a MS degree in Robotics and Intelligent Systems from Univ. Pierre et Marie Curie (Paris VI) in 2005. In 2012, he joined the FEMTOST Institute in the AS2M Department as Senior Scientist CNRS researcher (Besançon, FR). Currently, he works in microrobotics surgery and OCT (Optical Coherence Tomography)-based visual servoing.

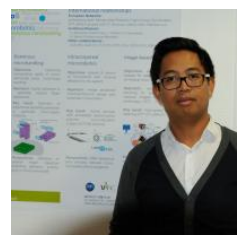

Kanty Rabenorosoa holds a MS degree (2007) in Electrical Engineering from INSA Strasbourg, FR and a Ph.D. in Automatic Control (2010) from Univ. de Franche-Comté, FR. He was a postdoc fellow at LIRMM (2011/2012) at Univ. of Montpellier, FR. He is currently Ass. Prof. at FEMTO-ST Institute in AS2M Dep. His research interests are microrobotic systems (e.g., Electro-Active Polymers (EAP) actuators, continuum robot, etc.) for medical applications within the MiNaRoB team.

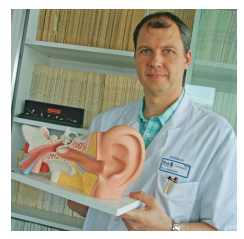

Laurent Tavernier is Professor and head of the ENT department of the CHRUB, since 2012. He is involved in several translational research projects dealing with the development of new tools and system for ENT minimally invasive surgery. Since 2009, Prof. Tavernier is the coordinator of the multicenter study financed by the Directorate General for the Provision Care (Ministry of Health) focusing in the prevention of post-operative bleeding risk in nasal endoscopy surgery. He is also member of the Society of Otolaryngology. French College of Otolaryngology, and of the French

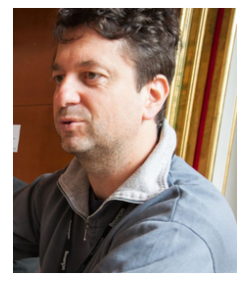

Nicolas Andreff received the Engineer degree (M.S. degree) in computer sciences and applied mathematics from ENSEEIHT, FR, in 1994, and the $\mathrm{Ph} . \mathrm{D}$. in computer vision, and robotics from INPG, Grenoble, FR, in 1999. He is currently a Prof. in the FEMTO-ST Institute, Univ. de Franche-Comté, FR $\mathrm{He}$ created the Biomedical Micro-/Nano-Robotics group in FEMTO-ST in 2012. His research interests range from visual servoing and parallel kinematics to intracorporeal microrobotics. 